\title{
Millimeter-Wave Compact and High-Performance Two-Dimensional Grid Array for 5G Applications
}

\author{
Syeda Fizzah Jilani ${ }^{1}$, Qammer H. Abassi ${ }^{2}$, and Akram Alomainy ${ }^{1}$ \\ ${ }^{1}$ School of Electronic Engineering and Computer Science, Queen Mary University of London, UK \\ ${ }^{2}$ School of Engineering, University of Glasgow, Glasgow G12 8QQ, UK \\ \{s.f.jilani, a.alomainy\}@qmul.ac.uk
}

\begin{abstract}
This paper presents the design and evaluation of a high gain, wideband, compact antenna grid array at millimeterwave (mm-wave) band for the 5 th generation (5G) applications. The designed microstrip-fed antenna array offers an operating bandwidth of $5.5 \mathrm{GHz}$ in the range of $27.5-33 \mathrm{GHz}$. The realized gain in the proposed range of operation is above $9 \mathrm{dBi}$ with a peak gain of $11.34 \mathrm{dBi}$ at $32.5 \mathrm{GHz}$. The numerically calculated efficiency is above $80 \%$ in the whole band of $5.5 \mathrm{GHz}$. The proposed antenna array is a potential candidate for the $5 \mathrm{G}$ cellular handheld devices as well as in the indoor base-stations.
\end{abstract}

Keywords-5G; antenna; circular; compact; grid; wireless.

\section{INTRODUCTION}

The recent advancement in wireless networks to ensure a high-speed and seamless communication, as well as the future demands, are expected to be limited by the issues of insufficient bandwidth at current sub-3 GHz spectrum. Future 5G systems and beyond are anticipated to be established on the mm-wave spectrum $[1,2]$. This transition is expected to deliver numerous advantages such as; shorter wavelengths to allow small form factor for the antennas, improved channel-width, wideband spread-spectrum ability, and choices of certain high-attenuation bands for highly secure point-to-point links [3, 4]. The unused $28 \mathrm{GHz}$ band has gained huge attention from the US Federal Communications Commission (FCC) to accommodate future $5 \mathrm{G}$ systems due to comparatively lower weather attenuations, lesser path loss and reduced signal fading [5]. The realization of $5 \mathrm{G}$ architecture necessitates sophisticated antenna designs to deal with the critical challenges of the unused mm-waves. A highly densified and short-range cell area is recommended with the execution of versatile indoor base-stations equipped with high-performance and compact antennas.

Simple geometries of patch antennas though offer planar integration, compact size, ease of fabrication and installation feasibility, and yet have limited gain and bandwidth. Bandwidth of the antenna can be improved with structural modifications, such as slots, fractals and monopole geometries, which are often associated with lower gain and efficiency [6]. Alternatively, the antenna gain can be enhanced by increasing the radiating area with parasitic patches or arrays, which typically compromises on compactness due to the extended size and holds limited bandwidth [7]. Recent research on $5 \mathrm{G}$ antennas has focused on achieving high gain and wide bandwidth [8-10]. Linear and grid arrays due to the compact size and high gain are favourable for $5 \mathrm{G}$, provided that bandwidth enhancement modifications are carried out [10-12]. This paper presents a high-performance array constituting a two-dimensional (2D) compact grid of circular patches. The proposed antenna operates at $28-\mathrm{GHz}$ with $5 \mathrm{G}$ features of high gain, low complexity, and wide bandwidth.

\section{ANTENNA ARRAY DESIGN AND FABRICATION}

The grid assembly for the designed antenna constitutes thirteen circular patches each with a radius of $0.175 \lambda_{o}$ (where wavelength, $\lambda_{o}=$ speed of light $(c) /$ resonant frequency $\left(f_{o}\right)$, estimated at $f_{o}=30 \mathrm{GHz}$ ). The antenna array is designed on the Rogers RT/Duroid $5880\left(\varepsilon_{\mathrm{r}}=2.2\right.$, and $\left.\tan \delta=0.0009\right)$. The array on a substrate of $21.5\left(2.15 \lambda_{o}\right) \times 23\left(2.3 \lambda_{o}\right) \times 0.8\left(0.08 \lambda_{o}\right) \mathrm{mm}^{3}$ dimensions, comprises of a continuous bottom ground plane of copper, while the top surface of the substrate includes the grid assembly provided with a feeding network. Microstrip feed line along with the stub of optimized dimensions is constructed for the impedance matching between the single feed point and the grid. The simulation modelling of the mm-wave antenna array, as well as the numerical evaluation of performance, is carried out in CST STUDIO SUITE software. The $50-\Omega$ matched $K$ connector is also designed in the simulation model to get the close approximation of the real-time measurement process. Fig. 1 (a) shows the designed antenna grid array with the optimized dimensions obtained after careful optimization. The fabrication of the designed antenna array is carried out by using LPKF milling machine. The substrate of Rogers RT/Duroid with a copper cladding thickness of $17.5 \mu \mathrm{m}$ on both sided is used. The prototyping is done on one side while the bottom metal layer remained intact as a continuous ground plane. Fig. 1 (b) shows the fabricated prototype of the proposed antenna.

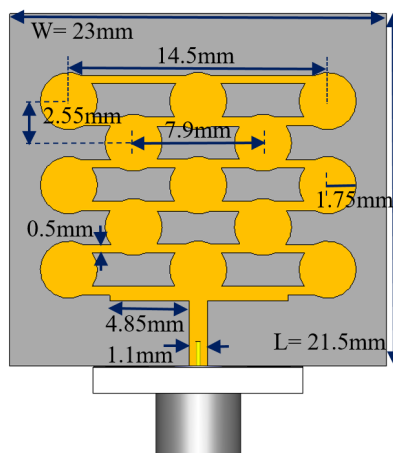

(a)

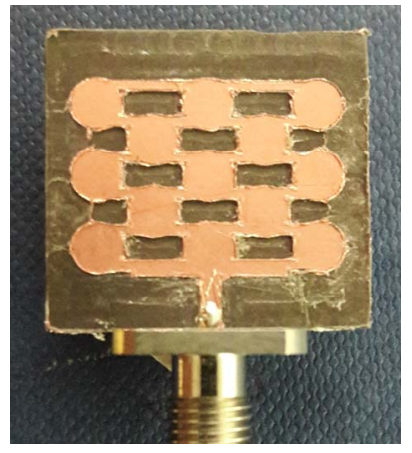

(b)
Fig. 1. The geomentry of the proposed grid antenna array for 5G; (a) simulated model with the optimized dimensions, (b) fabricated prototype. 


\section{NumericAl EVALUATION AND TESTING}

The performance of the designed 2-D antenna grid array is fully evaluated by parametric study and the numerical analysis in order to compute S-parameters, radiation pattern, and realized gain. Moreover, testing and measurements further validate the simulation findings. Fig. 2 illustrates the reflection coefficient $\left(\mathrm{S}_{11}\right)$ plots obtained in the simulation and testing of the antenna by the Vector Network Analyser (VNA). The simulation shows the bandwidth of 27.5-33.3 GHz, while the results of fabricated prototype testing cover a range of $27-33 \mathrm{GHz}$. This refers that the measurements of the array show good agreement with the simulated results in terms of antenna impedance bandwidth. Although, some mismatches have been observed mainly due to the fabrication intolerances, connector, and cable losses.

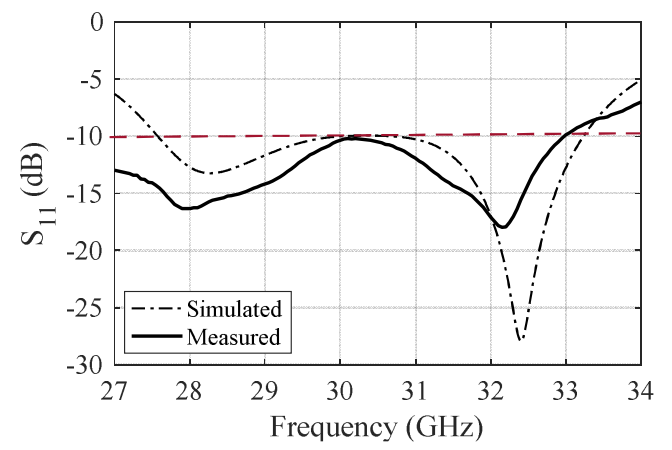

Fig. 2. Simulated and measured $\mathrm{S}_{11}$ of the proposed antenna grid array.

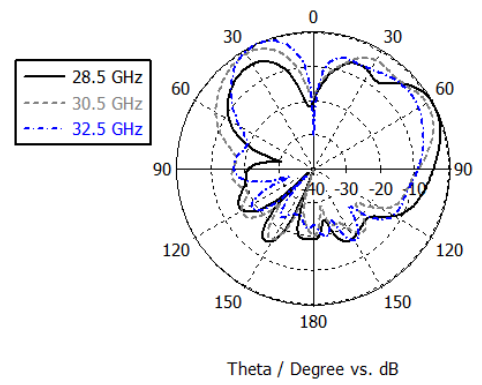

(a)

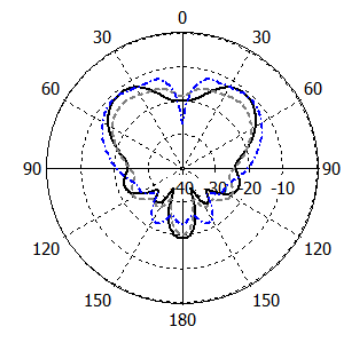

Theta / Degree vs. dB

(b)
Fig. 3. Numerically computed normalized radiation pattern of the proposed antenna grid array at three distinct frequencies: (a) E-plane cut at $\varphi=90^{\circ}$, (b) H-plane cut at $\varphi=0^{\circ}$.

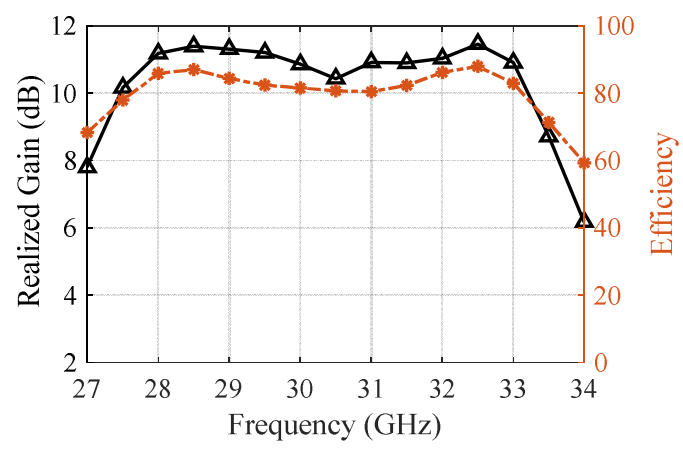

Fig. 4. Gain and efficiency vs. frequency of the proposed antenna grid array.
The radiation characteristics of the proposed grid antenna are shown in Fig. 3 for both E and H-plane cuts, normalized with the value of peak gain magnitude of $11.34 \mathrm{dBi}$. The plots of Fig. 3 (a) show that the antenna radiation is typically in the broadside direction, however split into two beams. While the side lobes magnitude is below $-10 \mathrm{~dB}$ as shown by Fig. 3 (b). Fig. 4 shows the realized gain and efficiency vs. frequency of the proposed antenna geometry over the operating range. The realized gain profile shows a magnitude above $9 \mathrm{dBi}$ in the $27.5-33 \mathrm{GHz}$ range with a peak gain of $11.34 \mathrm{dBi}$ at $32.5 \mathrm{GHz}$. While the total efficiency of the designed antenna computed in CST simulation is $80 \%$ or above in the desired range of operation.

\section{CONCLUSION}

This paper has presented a compact microstrip-fed grid antenna array for high gain and bandwidth demands in future cellular devices. The designed geometry has thirteen circular patch elements of the radius of $0.175 \lambda_{o}$, fitted in a compact area in a grid assembly. The grid array offers a bandwidth of 27.5$33 \mathrm{GHz}$ with the efficiency of above $80 \%$. The gain profile shows above $9 \mathrm{dBi}$ in the overall bandwidth with the peak gain of $11.34 \mathrm{dBi}$ at $32.5 \mathrm{GHz}$. The high performance of the designed antenna depicts its potential in the future $5 \mathrm{G}$ cellular devices.

\section{REFERENCES}

[1] D. Liu, W. Hong, T. S. Rappaport, C. Luxey and W. Hong, "What will 5G antennas and propagation be?," IEEE Trans. Antennas Propag., vol. 65 , no. 12, pp. 6205-6212, 2017.

[2] W. Hong, K. H. Baek and S. Ko, "Millimeter-wave 5G antennas for smartphones: Overview and experimental demonstration," IEEE Trans. Antennas Propag., vol. 65, no. 12, pp. 6250-6261, 2017.

[3] L. Wei, R. Q. Hu, Y. Qian, and G. Wu, "Key elements to enable millimeter wave communications for 5G wireless systems," IEEE Wireless Commun., vol. 21, no. 6, pp. 136-143, 2014.

[4] T. S. Rappaport, J. N. Murdock and F. Gutierrez, "State of the art in 60$\mathrm{GHz}$ integrated circuits and systems for wireless communications," Proc. IEEE, vol. 99, no. 8, pp. 1390-1436, 2011.

[5] Use of Spectrum Bands Above $24 \mathrm{GHz}$ for Mobile Radio Services, Federal Register, vol. 81, no. 219, Nov. 2016.

[6] S. Li, T. Chi, Y. Wang and H. Wang, "A millimeter-wave dual-feed square loop antenna for 5G communications," IEEE Trans. Antennas Propag., vol. 65, no. 12, pp. 6317-6328, 2017.

[7] H. A. Diawuo and Y. Jung, "Broadband proximity-coupled microstrip planar antenna array for 5G cellular applications," IEEE Antennas Wireless Propag. Lett., vol. 17, no. 7, pp. 1286-1290, 2018.

[8] S. F. Jilani, M. O. Munoz, Q. H. Abbasi and A. Alomainy, "Millimeterwave liquid crystal polymer based conformal antenna array for $5 \mathrm{G}$ applications," IEEE Antennas Wireless Propag. Lett., vol. 18, no. 1, pp. 84-88, 2019.

[9] A. Dadgarpour, M. Sharifi Sorkherizi and A. A. Kishk, "High-efficient circularly polarized magnetoelectric dipole antenna for $5 \mathrm{G}$ applications using dual-polarized split-ring resonator lens," IEEE Trans. Antennas Propag., vol. 65, no. 8, pp. 4263-4267, 2017.

[10] S. F. Jilani and A. Alomainy, "A multiband millimeter-wave 2-D array based on enhanced Franklin antenna for 5G wireless systems," IEEE Antennas Wireless Propag. Lett., vol. 16, pp. 2983-2986, 2017.

[11] Z. Chen and Y. P. Zhang, "FR4 PCB grid array antenna for millimeterwave $5 \mathrm{G}$ mobile communications," IEEE MTT-S Int. Microw. Workshop Series on RF and Wireless Tech. for Biomed. Healthcare Applications (IMWS-BIO), 2013, pp. 1-3.

[12] S. F. Jilani and A. Alomainy, "Millimeter-wave conformal antenna array for 5G wireless applications," IEEE Int. Symp. Antennas Propag. Society (APSURSI), 2017, pp. 1439-1440 